\title{
Softarisons: theory and practice
}

\author{
José Carlos R. Alcantud' ${ }^{1}$
}

Received: 28 January 2021 / Accepted: 26 June 2021 / Published online: 9 July 2021

(C) The Author(s) 2021

\begin{abstract}
This paper introduces the concept of softarison. Softarisons merge soft set theory with the theory of binary relations. Their purpose is the comparison of alternatives in a parameterized environment. We develop the basic theory and interpretations of softarisons. Then, the normative idea of 'optimal' alternatives is discussed in this context. We argue that the meaning of 'optimality' can be adjusted to fit in with the structure of each problem. A sufficient condition for the existence of an optimal alternative for unrestricted sets of alternatives is proven. This result means a counterpart of Weierstrass extreme value theorem for softarisons; thus, it links soft topology with the act of choice. We also provide a decision-making procedure-the minimax algorithm-when the alternatives are compared through a softarison. A case-study in the context of group interviews illustrates both the application of softarisons as an evaluation tool, and the computation of minimax solutions.
\end{abstract}

Keywords Soft set $\cdot$ Soft topology $\cdot$ Optimality $\cdot$ Multicriteria decision-making

\section{Introduction}

This article proposes a model that combines soft set theory with binary relations. The performance of binary relations is enhanced by the explanatory ability of soft set theory for vague intelligence. The gestalt they give rise to is called 'softarison'.

The goal of soft set theory [33] is to study imprecisely perceived knowledge about the performance of alternatives with respect to their descriptive attributes. Basically, a soft set provides a paramaterized description of the set of alternatives, the parameters being their fundamental characteristics. Some works $[9,13,27]$ have clarified the role of this theory among the wide realm of the theories of uncertainty. Others have established its basic operations (e.g., $[22,26,30,36])$. Textbooks summarize the fundamental elements of this theory [25]. In addition, direct extensions exist (incomplete soft sets [24,45] or $N$-soft sets [20]) and many hybrid models have been proposed too

José Carlos R. Alcantud

jcr@usal.es

1 BORDA Research Unit and Multidisciplinary Institute of Enterprise (IME), University of Salamanca,

37007 Salamanca, Spain (inclusive of fuzzy soft sets [29], hesitant fuzzy soft sets [16], Feng-rough soft set models [23], probabilistic soft sets [21], vague soft sets [42], et cetera; [28, 43] and [25, Chapter 5] reviewed several classes of hybrid soft set structures). Applications include medical diagnosis [32], forecasting [41] or data mining [35].

A first attempt to apply the core notion of soft set theory to binary relations has produced the successful concept of a soft relation [15]. Put succinctly, [15] defines a soft relation from a soft set to another soft set which consists of a soft subset of the product of both soft sets.

In continuation of this concern, in this article we investigate the problem from a novel perspective. Our position here is founded on a completely different origin. Softarisons are defined on a set of alternatives, rather than being defined between soft sets. Then, we take advantage of soft sets in order to establish parameterized comparisons between these alternatives. As is standard, the parameters correspond to the relevant attributes of the situation. This novel model appears to be natural and applicable since it uses the same primitive elements as standard soft set theory, namely, alternatives and their attributes, together with ordinary soft sets for this context.

We develop a basic theory of softarisons inclusive of their representations by tables. We make some consistency properties explicit. Then we explain that two semantics are 
available, and that this situation parallels the case of binary relations which are a building block of abstract decision theory. We also explore the meaning of 'optimal alternative' when the alternatives are compared through a softarison. Several criteria are put forward since the structure of the problem determines which elements can be used for differentiating the performance of the options. In particular, a sufficient condition for the existence of certain 'optimal' alternatives is proven without restrictions on cardinality. The condition is expressed in terms of soft compactness [14], a property defined for soft topological spaces $[6,19,37]$ that has received considerable attention [4], also in relation with soft topological ordered spaces [2].

The later achievement is a natural descendant of the Weierstrass extreme value theorem. This classical result gives a well-known sufficient condition for a function to attain a global minimum and a global maximum. Originally, it states that a continuous function defined on a closed bounded interval attains at least one global minimum and a global maximum. Its generalization to topological spaces requires the notion of a compact subset, and an appropriate generalization of the concept of continuity of a mapping between topological spaces. There are further extensions of the extreme value theorem. It can be improved so as to provide necessary and sufficient conditions for the existence of global minima, and of both global minima and maxima [31]. Its argument has been exported to maximization of binary relations, the most basic result being the Bergstrom-Walker theorem [17, 40]: it asserts that any continuous acyclic relation defined on a compact topological space has maximal elements. This theorem has also been extended to become a characterization [8]. In this paper, we produce an additional variation of the idea that continuity together with compactness ensure a suitable concept of maximality. We show that the argument is valid for a novel soft set based model for the expression of the preferences. Once again, 'compactness' (in fact, soft compactness) allows us to guarantee maximality in infinite universes (of alternatives). Therefore, in both cases, (soft) compactness allow ones to extend arguments from the finite to the infinite case.

It is remarkable that binary relations can also be merged with soft topological notions through soft topological ordered spaces [5] which are a recent concept reminiscent of topological ordered spaces [7, 34].

Finally, we adopt a procedural position in order to provide a decision-making algorithm for situations where a softarison captures the soft-theoretical comparisons between pairs of alternatives. This procedure relies on the idea of a minimax alternative. It is computed as the minimax of a proxy for the overall importance of the attributes for which an alternative outperforms another. We provide an illustrative case-study in the context of group interviews, a recruiting technique by which employers can simultaneously evaluate various applicants to a position.

The outline of this paper is as follows. Section 2 gives a concise and direct presentation of all necessary concepts from various areas (soft set theory, soft topology and binary relations). Section 3 introduces softarisons and discusses their representability and semantics. In Sect. 4 we link softarisons and choice from a normative perspective. Then, Sect. 5 produces a decision-making algorithm and our practical case. Section 6 gives further discussion about our novel tool. In addition, it points out at several lines for future research on this topic.

\section{Preliminary concepts}

This section contains some notions and terminology pertaining to soft set theory and binary relations $[8,38]$. We shall give all the prerequisites that we need in the rest of this article. Nevertheless, we avoid a lengthy and reiterative explanation of all the concepts and technical details, which can be easily found in related literature.

Throughout this paper $X$ denotes a non-empty set of alternatives. When $A$ is a set, its power set is denoted as $\mathcal{P}(A)$.

The formal definition of a soft set over the set (of alternatives) $X$ presumes the existence of another set of attributes $E$ that jointly characterize them:

Definition 2.1 [33] A soft set over the set $X$, is a pair $(F, E)$ where $F: E \rightarrow \mathcal{P}(X)$.

Henceforth, $S S_{E}(X)$, or simply $S S(X)$ when $E$ is common knowledge, represents the set of all soft sets over $X$ with attributes $E$.

As is well known, the interpretation of this structure is that for each $e \in E, F(e)$ represents the set of alternatives from $X$ that satisfy the property represented by $e \in E$.

The operations with soft sets are performed in a more convenient manner with the help of two possible descriptions:

(1) Any soft set $(F, E)$ can be summarized by the notation $\{(e, F(e)): e \in E\}$.

(2) In the event that both $X$ and $E$ are finite, the soft set $(F, E)$ can be represented in tabular form too. Figure 1 shows this representation when $X=$ $\left\{o_{1}, o_{2}, \ldots, o_{m}\right\}$ and $E=\left\{e_{1}, e_{2}, \ldots, e_{n}\right\}$. A convention is made that we write $r_{j k}=1$ when $o_{j} \in F\left(e_{k}\right)$, and we write $r_{j k}=0$ otherwise. 


\begin{tabular}{ccccc}
$(F, E)$ & $e_{1}$ & $e_{2}$ & $\cdots$ & $e_{n}$ \\
\hline$O_{1}$ & $r_{11}$ & $r_{12}$ & $\cdots$ & $r_{1 n}$ \\
$O_{2}$ & $r_{21}$ & $r_{22}$ & $\cdots$ & $r_{2 n}$ \\
$\vdots$ & $\vdots$ & $\vdots$ & & $\vdots$ \\
$o_{m}$ & $r_{m 1}$ & $r_{m 2}$ & $\cdots$ & $r_{m n}$
\end{tabular}

Fig. 1 Tabular representation of a soft set $(F, E)$ when the sets of alternatives and attributes are finite

Two special soft sets are the null $\Phi$ and the absolute $\tilde{X}$ soft sets over $X$. They are such that $\Phi(e)=\varnothing$ for each $e \in E$, and $\tilde{X}(e)=X$ for each $e \in E$.

Intersections and unions, as well as inclusions, can be defined on soft sets [30]. Suppose $\left(F_{1}, E\right),\left(F_{2}, E\right)$ $\in S_{E}(X)$. Their soft union is denoted as $\left(F_{1}, E\right) \sqcup\left(F_{2}, E\right)$. It is $(F, E) \in S S_{E}(X)$ such that $F(e)=F_{1}(e) \cup F_{2}(e)$ for each $e \in E$. Their soft intersection is denoted as $\left(F_{1}, E\right) \sqcap\left(F_{2}, E\right)$. It is $\left(F^{\prime}, E\right) \in S S_{E}(X)$ such that $F^{\prime}(e)=$ $F_{1}(e) \cap F_{2}(e)$ for each $e \in E$. The extension of these operations to infinite collections of soft sets over a common set are immediate.

As to soft inclusion, we denote it as $\left(F_{1}, E\right) \sqsubseteq\left(F_{2}, E\right)$, and it holds when $F_{1}(e) \subseteq F_{2}(e)$ for each $e \in E$. Note that alternative definitions of 'soft subsethood' an 'soft equality' exist $[22,26,36]$.

We are now ready to recall the concept of a soft topology:

Definition 2.2 $[19,37]$ A soft topology on $X$ is a collection of soft sets over $X$, i.e., $\tau \subseteq \mathcal{P}\left(S S_{E}(X)\right)$ that satisfies the properties:

(1) $\Phi, \tilde{X} \in \tau$;

(2) the soft union of any number of soft sets from $\tau$ belongs to $\tau$; and

(3) the soft intersection of any finite number of soft sets from $\tau$ belongs to $\tau$.

Soft topologies have been studied extensively $[2-4,6,10,14,39,44]$. Their elements are called soft open sets. Notice that just like the open sets in a point-set topology are sets, the soft open sets in soft topologies are soft sets.

We shall need the notion of soft compactness of a soft topology, that in turn uses the concept of cover of $(F, E) \in S S_{E}(X)$ : it is a collection $\Psi$ of soft sets over $X$ such that $(F, E) \sqsubseteq \sqcup\left\{\left(F^{\prime}, E\right):\left(F^{\prime}, E\right) \in \Psi\right\}$. When all the soft sets in $\Psi$ are soft open, we say that $\Psi$ is a soft open cover of $(F, E)$. Also, a subcover of $\Psi$ is a subcollection of elements from $\Psi$ that is also a cover.
Definition $2.3[14,44]$ A soft topology $\tau$ on $X$ is soft compact if every soft open cover of $\tilde{X}$ has a finite subcover.

Now, we recall the following concepts that concern binary relations.

Definition 2.4 Assume that $X$ is a set. Then, a binary relation $R$ on $X$ is a subset of the Cartesian product $X \times X$. We interpret $(x, y) \in X \times X$ as ' $x$ is related to $y$ ' and we often denote it as $x R y$ for convenience.

Binary relations are a standard model for the representation of the preferences of the agents. They allow us to capture two different types of semantics which determine the presentation of subsequent concepts:

(1) Under the weak semantics of $R$, we assume that $x R y$ represents the broad idea that ' $x$ is at least as good as $y$ '.

(2) Under the strict semantics of $R$, we assume that $x R y$ means that ' $x$ is strictly better than $y$ '.

It is very important to note which meaning is attached to any binary relation we work with. Properties that are natural for the weak semantics are undesirable in the strict interpretation (like in the case of reflexivity), and conversely (irreflexivity). Also certain ideas should be associated with finely tuned concepts. Greatest elements are the adequate concept for the idea of 'a best alternative' under the weak semantics (usually captured by reflexive relations). However maximal elements better express the idea of 'a best alternative' under the strict semantics (usually captured by asymmetric or irreflexive relations). We proceed to recall both concepts:

Definition 2.5 [38, (A.11), (A.13)] Suppose that $R$ is a binary relation on a set (of alternatives) $X$. We say that $y \in X$ is an $R$-greatest (or simply greatest) alternative if $y R x$ for each $x \in X$. And we say that $y \in X$ is $R$-maximal (or simply maximal) if for each $x \in X, x R y$ is false.

The reader is addressed to $[12,18,38]$ for other fundamental definitions about binary relations.

Our next section introduces a model that blends soft set theory with binary relations. Their combined features produce 'softarisons' which to some extent, reproduce the benefits of binary relations while enhancing them with the descriptive power of soft set theory.

\section{Softarison: a soft-set-based methodology for making comparisons}

As a first point, this section defines softarisons. Then, we expound the semantics of this new concept and give some illustrative examples in the finite case. We also link them to a family of binary relations indexed by the attribute set. 
Henceforth, in this section, $X$ denotes a (possibly infinite) non-empty set of alternatives, and $E$ is a (possibly infinite) set of attributes.

Definition 3.1 An $E$-softarison over $X$ is a collection of soft sets over $X$ indexed by $X$. Formally:

$\mathcal{S}=\left\{\left(F^{x}, E\right)\right\}_{x \in X}$ such that $\left(F^{x}, E\right) \in S S_{E}(X)$ for each

$$
\in X \text {. }
$$

Or alternatively, an $E$-softarison over $X$ is a mapping

$\mathcal{S}: X \longrightarrow S S_{E}(X)$.

We simply refer to softarisons when $E$ is common knowledge.

Softarison is an acronym for soft-comparison. Indeed, for the purpose of making effective use of this concept we interpret: when $x \in X$ and $e \in E$,

$y \in F^{x}(e)$ if and only if $x$ is preferred to

$y$ in terms of attribute $e$.

Therefore, when we have a softarison $\mathcal{S}=\left\{\left(F^{x}, E\right)\right\}_{x \in X}$, a soft set $\left(F^{x}, E\right)$ encapsulates the benefits of option $x$ with respect to every other alternative, when each attribute is considered separately.

We now proceed to discuss the structure of the finite case and then we shall give illustrative examples with their natural interpretations. All this is done in Sect. 3.1. We relate this model to binary relations in Sect. 3.2. Afterward in Sect. 3.3, we present some properties of consistency of softarisons. We postpone a more detailed discussion of the semantics of softarisons till Sect. 3.4.

\subsection{Softarisons for finite settings}

When the set of alternatives is finite, say $X=\left\{o_{1}, \ldots, o_{m}\right\}$, a softarison $\mathcal{S}=\left\{\left(F^{x}, E\right)\right\}_{x \in X}$ becomes $\mathcal{S}=\left\{\left(F^{o_{1}}, E\right), \ldots\right.$, $\left.\left(F^{o_{m}}, E\right)\right\}$. Each of these soft sets can be expressed in tabular form when the set of attributes is finite too. Therefore, in this case, a softarison is a list of $m$ tables, one corresponding with each alternative. Figure 2 displays the general structure of this information. In this representation,

$r_{j}^{i}\left(e_{k}\right)=\left\{\begin{array}{lc}1, & \text { if } o_{j} \in F^{o_{i}}\left(e_{k}\right), \\ 0, & \text { otherwise }\end{array}\right.$

Examples 3.2 and 3.3 below illustrate Definition 3.1 in this context.

Example 3.2 Let $X=\left\{o_{1}, o_{2}, o_{3}\right\}$ and $E=\left\{e_{1}, e_{2}, e_{3}\right\}$. In order to establish the comparative performance of the alternatives in $X$ with respect to the properties in $E$, a softarison $\mathcal{S}^{\prime}=\left\{\left(F^{o_{1}}, E\right),\left(F^{o_{2}}, E\right),\left(F^{o_{3}}, E\right)\right\}$ is given. Figure 3 displays such a softarison in tabular form.

According to Equations (3) and (4), we interpret that alternative $o_{1}$ is preferred to $o_{2}$ if we only consider attribute $e_{1}$, and it is preferred to $o_{3}$ if we only consider attribute $e_{3}$. No further preferences of $o_{1}$ over other alternatives are apparent from the first table. If we now look at the second table, we observe that $o_{2}$ is preferred to $o_{3}$ if we only consider either attribute $e_{1}$ or attribute $e_{2}$. Finally, the third table claims that $o_{3}$ is preferred to $o_{1}$ if we only consider attribute $e_{2}$, and it is preferred to $o_{2}$ if we only consider attribute $e_{3}$.

Example 3.3 In the situation of Example 3.2, Figure 4 displays another softarison $\mathcal{S}^{\prime \prime}=\left\{\left(H^{o_{1}}, E\right),\left(H^{o_{2}}, E\right)\right.$, $\left.\left(H^{o_{3}}, E\right)\right\}$ over $X$ in tabular form.

According to this softarison over $X$, the first table captures the fact that alternative $o_{1}$ is preferred to $o_{2}$ if we consider either attribute $e_{1}$ or attribute $e_{3}$. No further preference of $o_{1}$ over other alternatives is put forward. The second table shows that $o_{2}$ is preferred to $o_{3}$ if we only consider either attribute $e_{1}$ or attribute $e_{2}$. Finally, the third table claims that $o_{3}$ is preferred to $o_{1}$ if we only consider either attribute $e_{2}$ or attribute $e_{3}$.

\begin{tabular}{ccccc}
$\left(F^{o_{m}}, E\right)$ & $e_{1}$ & $e_{2}$ & $\cdots$ & $e_{n}$ \\
\hline$o_{1}$ & $r_{1}^{m}\left(e_{1}\right)$ & $r_{1}^{m}\left(e_{2}\right)$ & $\cdots$ & $r_{1}^{m}\left(e_{n}\right)$ \\
$o_{2}$ & $r_{2}^{m}\left(e_{1}\right)$ & $r_{2}^{m}\left(e_{2}\right)$ & $\cdots$ & $r_{2}^{m}\left(e_{n}\right)$ \\
$\vdots$ & $\vdots$ & $\vdots$ & & $\vdots$ \\
$o_{m}$ & $r_{m}^{m}\left(e_{1}\right)$ & $r_{m}^{m}\left(e_{2}\right)$ & $\cdots$ & $r_{m}^{m}\left(e_{n}\right)$
\end{tabular}

Fig. 2 Tabular representation of a softarison when the sets of alternatives and attributes are finite. Equations (3) and (4) explain the convention for the figures in these tables 


\begin{tabular}{|c|c|c|c|c|c|c|c|c|c|c|c|}
\hline$\left(F^{o_{1}}, E\right)$ & $e_{1}$ & $e_{2}$ & $e_{3}$ & $\left(F^{o_{2}}, E\right)$ & $e_{1}$ & $e_{2}$ & $e_{3}$ & $\left(F^{o_{3}}, E\right)$ & $e_{1}$ & $e_{2}$ & $e_{3}$ \\
\hline$O_{1}$ & 0 & 0 & 0 & $O_{1}$ & 0 & 0 & 0 & $o_{1}$ & 0 & 1 & 0 \\
\hline $\mathrm{O}_{2}$ & 1 & 0 & 0 & $\mathrm{O}_{2}$ & 0 & 0 & 0 & $\mathrm{O}_{2}$ & 0 & 0 & 1 \\
\hline$O_{3}$ & 0 & 0 & 1 & $O_{3}$ & 1 & 1 & 0 & $O_{3}$ & 0 & 0 & 0 \\
\hline
\end{tabular}

Fig. 3 Tabular representation of the softarison $\mathcal{S}^{\prime}=\left\{\left(F^{o_{1}}, E\right),\left(F^{o_{2}}, E\right),\left(F^{o_{3}}, E\right)\right\}$ over $X$ in Example 3.2

\begin{tabular}{cccc}
$\left(H^{o_{1}}, E\right)$ & $e_{1}$ & $e_{2}$ & $e_{3}$ \\
\hline$o_{1}$ & 0 & 0 & 0 \\
$o_{2}$ & 1 & 0 & 1 \\
$o_{3}$ & 0 & 0 & 0
\end{tabular}

\begin{tabular}{cccc}
$\left(H^{o_{2}}, E\right)$ & $e_{1}$ & $e_{2}$ & $e_{3}$ \\
\hline$o_{1}$ & 0 & 0 & 0 \\
$o_{2}$ & 0 & 0 & 0 \\
$o_{3}$ & 1 & 1 & 0
\end{tabular}

\begin{tabular}{cccc}
$\left(H^{o_{3}}, E\right)$ & $e_{1}$ & $e_{2}$ & $e_{3}$ \\
\hline$o_{1}$ & 0 & 1 & 1 \\
$o_{2}$ & 0 & 0 & 0 \\
$o_{3}$ & 0 & 0 & 0
\end{tabular}

Fig. 4 Tabular representation of the softarison $\mathcal{S}^{\prime \prime}=\left\{\left(H^{o_{1}}, E\right),\left(H^{o_{2}}, E\right),\left(H^{o_{3}}, E\right)\right\}$ in Example 3.3

\subsection{Binary relations associated with a softarison}

We proceed to show that an $E$-softarison $\left\{\left(F^{x}, E\right)\right\}_{x \in X}$ over $X$ induces a family $\left\{R_{e}\right\}_{e \in E}$ of binary relations on $X$, indexed by $E$.

To do that, each attribute $e \in E$ induces a binary relation $R_{e}$ on $X$ according to the following rule:

$x R_{e} y$ if and only if $y \in F^{x}(e)$

This definition is the natural interpretation of softarisons expressed by Equation (3): it establishes $x R_{e} y$ if and only if $x$ is preferred to $y$ in terms of attribute $e$.

When a tabular representation in the format of Figure 2 exists for a softarison over the set $X$, we read that for each $o_{i}, o_{j} \in X$ and $e_{k} \in E: o_{i} R_{e_{k}} o_{j}$ if and only if $r_{j}^{i}\left(e_{k}\right)=1$.

Example 3.4 In the situation of Example 3.2, three binary relations $\left\{R_{e_{1}}, R_{e_{2}}, R_{e_{3}}\right\}$ are derived from the data by Equation (5), namely

$$
\begin{aligned}
& o_{1} R_{e_{1}} o_{2} R_{e_{1}} o_{3}, \\
& o_{2} R_{e_{2}} o_{3} R_{e_{2}} o_{1}, \\
& o_{1} R_{e_{3}} o_{3} R_{e_{3}} o_{2} .
\end{aligned}
$$

Example 3.5 In the situation of Example 3.3, three binary relations $\left\{R_{e_{1}}^{\prime}, R_{e_{2}}^{\prime}, R_{e_{3}}^{\prime}\right\}$ are derived:

$$
\begin{aligned}
& o_{1} R_{e_{1}}^{\prime} o_{2} R_{e_{1}}^{\prime} o_{3}, \\
& o_{2} R_{e_{2}}^{\prime} o_{3} R_{e_{2}}^{\prime} o_{1}, \\
& o_{3} R_{e_{3}}^{\prime} o_{1} R_{e_{3}}^{\prime} o_{2} .
\end{aligned}
$$

The construction in this section creates a link between softarisons and another tool for the expression of preferences. Indeed the literature on binary relations is tremendously large. Their role in decision-making, mathematical economics, mathematical psychology, and many other branches is of paramount importance [12,38]. Thus this literature is a good source for inspiration.

The next section will take advantage of the existence of associated binary relations in order to transfer some properties of consistency from binary relations to softarisons. We expound how some can be tested by their tabular representations easily, provided that the setting is finite.

\subsection{Consistency properties of softarisons}

Not all sets of data are sufficiently reliable, and special care must be taken to jump to conclusions if they arise from subjective estimates. When we use data that produce comparisons, it is particularly important to verify what consistency qualities they satisfy. This is regardless of the framework where comparisons are established. For example, monographs have been written on rationality or consistency properties of comparisons given by binary relations $[12,18,38]$.

Relatedly, Sect. 3.2 gives a simple way to define some consistency properties of softarisons: We can do that in terms of its associated family of binary relations. Therefore, let $\left\{R_{e}\right\}_{e \in E}$ be defined from the $E$-softarison $\mathcal{S}=$ $\left\{\left(F^{x}, E\right)\right\}_{x \in X}$ over $X$ by Equation (5). Consider any property of binary relations $P$ (for example, reflexivity, asymmetry, transitivity, ....) We say that the $E$-softarison is $P$ when $R_{e}$ satisfies $P$ for each $e \in E$. And we say that it is pre- $P$ when $R_{e}$ satisfies $P$ for some $e \in E$.

Thus for example, henceforth we say that an $E$-softarison is reflexive when each $R_{e}$ is reflexive. We say that it is irreflexive when each $R_{e}$ is irreflexive. And it is asymmetric when each $R_{e}$ is asymmetric. Likewise, an $E$-softarison is pre-transitive if at least one of the $R_{e}$ relations is transitive; and it is pre-acyclic when at least one $R_{e}$ relation is acyclic.

Let us illustrate these definitions:

Example 3.6 By Example 3.4, the softarison defined in Example 3.2 is irreflexive and acyclic (therefore 
asymmetric); however, it is not even pre-transitive (neither of the three relations derived from it is transitive).

The same is true for the softarison defined in Example 3.3, by Example 3.5.

Let us write down explicit definitions of the most fundamental concepts that we shall use in the next sections. An $E$-softarison $\mathcal{S}$ over $X$ is:

- Reflexive when $x \in F^{x}(e)$, for each $x \in X$ and $e \in E$.

- Irreflexive when $x \notin F^{x}(e)$, for each $x \in X$ and $e \in E$.

- Asymmetric when $x \in F^{y}(e)$ implies $y \notin F^{x}(e)$, for each $x, y \in X$ and $e \in E$.

In the finite setting studied in Sect. 3.1, we do not need to actually write down the binary relations associated with a softarison in order to study all their consistency properties. They should be apparent from the tabular representation of the softarison. The aforementioned three properties are especially simple to check:

- Reflexive softarisons are characterized by tabular representations with $r_{i}^{i}\left(e_{k}\right)=1$ for each $i \in\{1, \ldots, m\}$ and $k \in\{1, \ldots, n\}$. In other words: row $i$ of table $i$ is filled with ones, for each $i$.

- Irreflexive softarisons are characterized by tabular representations with $r_{i}^{i}\left(e_{k}\right)=0$ for each $i \in\{1, \ldots, m\}$ and $k \in\{1, \ldots, n\}$. In other words: row $i$ of table $i$ is filled with zeros, for each $i$.

- Asymmetric softarisons are characterized by tabular representations that satisfy: $r_{i}^{j}\left(e_{k}\right)=1$ implies $r_{j}^{i}\left(e_{k}\right)$ $=0$, for each $i, j \in\{1, \ldots, m\}$ and $k \in\{1, \ldots, n\}$. In other words: when there is a 1 at row $i$ and column $k$ of table $j$, there is a 0 at row $j$ and column $k$ of table $i$, for each feasible $i, j, k$.

\subsection{Weak and strict semantics of softarisons}

We are now ready to explain the semantics of softarisons in more detail. Earlier in this section we submitted the general idea that a softarison is a 'soft comparison'. By this, we meant that for each alternative and attribute, it produces the set of elements for which the alternative is preferred to them with regard to that attribute. However the term 'preferred' is deliberately ambiguous. It admits two different semantics:

(1) The weak semantics is related to reflexive softarisons.

Here 'preferred' means 'at least as good as'. For this reason, $x \in F^{x}(e)$ must always be true: no matter what property we focus on, any alternative is 'at least as good as' itself in terms of that property.

In this semantics, it is possible that both $x \in F^{y}(e)$ and $y \in F^{x}(e)$ hold true. This case is associated with indifference or indecisiveness: we are declaring that when we only consider attribute $e, y$ is at least as good as $x$, and $x$ is at least as good as $y$.

(2) The strict semantics is related to irreflexive softarisons.

Here 'preferred' means 'strictly better than'. For this reason, $x \in F^{x}(e)$ is never true: for each property we focus on, an alternative is never 'strictly better than' itself in terms of that property.

The classification above is the natural replication of the discussion given for binary relations in Sect. 2. Obviously it relies on very basic consistency requirements. In the strict semantics, one would at least expect asymmetric softarisons from a consistent evaluation. For if asymmetry is contradicted we would be admitting that an alternative is 'strictly better than' another alternative which in turn, is 'strictly better than' the first one, when both comparisons are made by focusing on the same attribute.

Remark 3.7 Neither of these semantics is superior to the other. The practitioner must decide what type of evaluations will be used and proceed accordingly. If the opinion of an expert is required, he/she should be instructed to either declare which options are 'at least as good as' others, or alternatively, he/she must declare which options are 'strictly better' than others. All evaluations must be made separately in consideration of each attribute.

We are now enabled to discuss the idea of an 'optimal alternative' in the framework that we have designed. The next section discusses this important issue.

\section{The selection of optimal alternatives: a normative analysis}

We cannot disentangle soft sets (or soft-set-based theories) from multi-criteria decision-making: soft sets are defined from multiple attributes. This poses a difficulty for deciding what alternative is 'optimal' since we are obliged to balance the often opposed recommendations of the attributes. Alternatives that are excellent in terms of an attribute may be dreadful in relation with other attributes.

In the face of this problem, two positions may be taken:

Normative theories attempt to define the characteristics that an 'optimal' alternative should meet. The downside of this approach is that not always such 'optimal' alternatives exist; and in addition, different concepts may be arguably 'optimal'. A fundamental line of research investigates the conditions that guarantee or characterize the existence of 'optimal' alternatives, whatever the exact meaning of this term. 
Procedural decision-making bypasses this discussion. It concentrates on producing one (possibly multiple) output. Quite often, the procedure that gives the solution is an algorithm.

In this Section, we adopt a normative position in order to improve the theoretical foundations of softarions. We proceed to discuss some concepts that are arguably acceptable as an 'optimal' solution, in several frameworks. Then Sect. 5 gives a discussion about a procedural approach to decision-making based on softarisons with an example.

\subsection{The choice of optimal alternatives when $E$ is finite}

Along this section, we assume that $E=\left\{e_{1}, \ldots, e_{m}\right\}$ is finite and $\mathcal{S}$ is a softarison over $X$. No other special assumptions on $\mathcal{S}$ are made. In particular, $X$ may or may not be infinite.

The next concept will be used as a proxy of the performance of the alternatives:

Definition 4.1 The inverse score of $x$ respect to $y$ is $I^{y}(x)=\left|\left\{e \in E \mid x \in F^{y}(e)\right\}\right|$. When $X=\left\{o_{1}, \ldots, o_{n}\right\}$, the inverse score of $o_{i}$ respect to $o_{j}$ is also written as

$I^{o_{j}}\left(o_{i}\right)=\left|\left\{e \in E \mid o_{i} \in F^{o_{j}}(e)\right\}\right|=\sum_{k=1}^{n} r_{i}^{j}\left(e_{k}\right)$

The inverse score $I^{y}(x)$ captures the number of attributes for which $y$ performs better than $x$. If a tabular representation is available because $X=\left\{o_{1}, \ldots, o_{n}\right\}$ is finite, $I^{o_{j}}\left(o_{i}\right)$ is the sum of the indices in row $i$ of the table associated with $o_{j}$.

Remark 4.2 It is just natural to generalize the concept introduced in Definition 4.1 to a 'weighted' version, provided that we have weights associated with the attributes in $E$. We just need to modify the expressions in Definition 4.1 as follows. When $\omega=\left(\omega_{1}, \ldots, \omega_{m}\right)$ is a vector of weights with $\omega_{1}+\ldots+\omega_{n}=1, \omega_{i} \in[0,1]$ for each $i=1, \ldots, n$, then $W_{\omega}^{y}(x)=\sum_{k=1}^{n}\left\{\omega_{k} \mid x \in F^{y}\left(e_{k}\right)\right\}$ is the $\omega$-weighted inverse score of $x$ respect to $y$. It gives us a measure of the total weight (instead of the total number) of the attributes for which $y$ performs better than $x$.

When $X=\left\{o_{1}, \ldots, o_{n}\right\}$, the $\omega$-weighted inverse score of $o_{i}$ respect to $o_{j}$ is written as

$W_{\omega}^{o_{j}}\left(o_{i}\right)=\sum_{k=1}^{n} \omega_{k} r_{i}^{j}\left(e_{k}\right)$.
In the current context, we submit that alternatives that are never outperformed in terms of inverse scores are especially interesting. In formal terms, an appealing solution should be an alternative that satisfies the following property:

Definition 4.3 The alternative $y \in X$ is score undominated if

$I^{y}(x) \geqslant I^{x}(y)$ for each $x \in X$.

If a vector of weights $\omega$ for the attributes exists, $y$ is weighted-score undominated if

$W_{\omega}^{y}(x) \geqslant W_{\omega}^{x}(y)$ for each $x \in X$

In words, score undominated alternatives satisfy the following property: when they are compared with any other alternative, the number of attributes for which the first improves the second, is at least as high as the number of attributes for which the second improves the first. Score undominated options beat any other alternative in pairwise comparisons, when this comparison is made by the number of attributes for which one improves the other. The weighted version of this concept replaces 'number of attributes' by 'overall weight of the attributes' in this explanation.

It is not true that all softarisons have score undominated alternatives:

Example 4.4 The softarison $\mathcal{S}^{\prime}$ in Example 3.2 has one score undominated alternative, namely, $o_{1}$. Figure 5 displays the inverse scores associated with the softarison defined in this example. Notice that $I^{o_{1}}\left(o_{2}\right)=$ $1>I^{o_{2}}\left(o_{1}\right)=0$ and $I^{o_{1}}\left(o_{3}\right)=1=I^{o_{3}}\left(o_{1}\right)$. However, $o_{2}$ does not satisfy this property: $I^{o_{2}}\left(o_{1}\right)=0<I^{o_{1}}\left(o_{2}\right)=1$ as mentioned above. And $o_{3}$ does not satisfy Definition 4.3 either: $I^{o_{3}}\left(o_{2}\right)=1<I^{o_{2}}\left(o_{3}\right)=2$.

By contrast the softarison $\mathcal{S}^{\prime \prime}$ defined in Example 3.3, whose inverse scores are given in Fig. 6, lacks score undominated alternatives. The fact $I^{o_{3}}\left(o_{1}\right)=2>I^{o_{1}}\left(o_{3}\right)=$ 0 discards $o_{1}, I^{o_{1}}\left(o_{2}\right)=2>I^{o_{2}}\left(o_{1}\right)=0$ discards $o_{2}$, and $I^{o_{2}}\left(o_{3}\right)=2>I^{o_{3}}\left(o_{2}\right)=0$ discards $o_{3}$.

Example 4.4 raises a natural question: which properties of a softarison ensure that score undominated alternatives

\begin{tabular}{c|ccc} 
& $o_{1}$ & $o_{2}$ & $o_{3}$ \\
\hline$o_{1}$ & $I^{o_{1}}\left(o_{1}\right)=0$ & $I^{o_{2}}\left(o_{1}\right)=0$ & $I^{o_{3}}\left(o_{1}\right)=1$ \\
$o_{2}$ & $I^{o_{1}}\left(o_{2}\right)=1$ & $I^{o_{2}}\left(o_{2}\right)=0$ & $I^{o_{3}}\left(o_{2}\right)=1$ \\
$o_{3}$ & $I^{o_{1}}\left(o_{3}\right)=1$ & $I^{o_{2}}\left(o_{3}\right)=2$ & $I^{o_{3}}\left(o_{3}\right)=0$
\end{tabular}

Fig. 5 Inverse scores of the softarison $\mathcal{S}^{\prime}$ defined in Example 3.2 


$$
\begin{array}{c|ccc} 
& o_{1} & o_{2} & o_{3} \\
\hline o_{1} & I^{o_{1}}\left(o_{1}\right)=0 & I^{o_{2}}\left(o_{1}\right)=0 & I^{o_{3}}\left(o_{1}\right)=2 \\
o_{2} & I^{o_{1}}\left(o_{2}\right)=2 & I^{o_{2}}\left(o_{2}\right)=0 & I^{o_{3}}\left(o_{2}\right)=0 \\
o_{3} & I^{o_{1}}\left(o_{3}\right)=0 & I^{o_{2}}\left(o_{3}\right)=2 & I^{o_{3}}\left(o_{3}\right)=0
\end{array}
$$

Fig. 6 Inverse scores of the softarison $\mathcal{S}^{\prime \prime}$ defined in Example 3.3

exist? We first propose two sufficient conditions that are rather naive and easy to check, and then we put forward a more demanding condition.

The first one is quite obvious:

Proposition 4.5 If $y \in X$ satisfies $I^{x}(y)=0$ for all $x \in X$, then $y$ is score undominated. If a vector of weights $\omega$ for the attributes exists, $y$ is weighted-score undominated when $W_{\omega}^{x}(y)=0$ for each $x \in X$.

Notice that any alternative $y$ that satisfies the assumption of this Proposition must also satisfy $W_{\omega}^{y}(x) \geqslant W_{\omega}^{x}(y)=0$ for each $x \in X$. This obvious fact guarantees that $y$ is weighted-score undominated.

Remark 4.6 Corollary 4.12 below relates Proposition 4.5 to a different concept of optimality.

Now we prove a less trivial sufficient condition for the existence of score undominated alternatives:

Proposition 4.7 Suppose $\mathcal{S}$ is an asymmetric softarison over X. If $y \in X$ satisfies $I^{y}(x) \geqslant \frac{|E|}{2}$ for all $x \in X$, then $y$ is score undominated.

Proof Let us fix an arbitrary $o_{i} \in X$. By Definition 4.1, $I^{y}(x)=\left|\left\{e \in E \mid x \in F^{y}(e)\right\}\right|$. Because the softarison is asymmetric, $I^{x}(y)=\left|\left\{e \in E \mid y \in F^{x}(e)\right\}\right| \leqslant \frac{|E|}{2} \leqslant I^{y}(x)$.

This proves that $y \in X$ satisfies Definition 4.3.

Example 4.4 proves that neither of the sufficient conditions in Propositions 4.5 and 4.7 are necessary. It is therefore important to give other sufficient conditions for the existence of score undominated alternatives. We proceed to state another more technical solution to this problem.

\subsection{The choice of optimal alternatives when $E$ and $X$ are finite}

Section 3.2 produced a collection of binary relations for any softarison (one for each attribute). With the aid of the concepts in this section, we now define a new binary relation associated with any softarison when $E$ is finite:

Definition 4.8 For each $x, y \in X$, $x \succ_{\mathcal{S}} y$ if and only if $I^{x}(y)>I^{y}(x)$.

Example 4.4 computed the inverse scores from the softarison $\mathcal{S}^{\prime \prime}$ defined in Example 3.3. One can observe $o_{1} \succ_{\mathcal{S}^{\prime \prime}} O_{2} \succ_{\mathcal{S}^{\prime \prime}} O_{3} \succ_{\mathcal{S}^{\prime \prime}} O_{1}$, so $\mathcal{S}^{\prime \prime}$ has a cycle. We proceed to show that this may be the technical reason for the absence of score undominated alternatives for $\mathcal{S}^{\prime \prime}$. The next formal definition is needed:

Definition 4.9 We say that $\mathcal{S}$ is score-acyclic when $\succ_{\mathcal{S}}$ is acyclic.

We are now ready to prove a sufficient condition for the existence of score undominated alternatives when both $E$ and $X$ are finite:

Proposition 4.10 Suppose $\mathcal{S}$ is a softarison over X, which is finite. If $\mathcal{S}$ is score-acyclic then there is a score undominated alternative for $\mathcal{S}$.

Proof It is well known that there is a maximal element of $\succ_{\mathcal{S}}$ in $X$ [38, Theorem A(3)]. Therefore there is $y \in X$ such that for each $x \in X, x \succ \mathcal{S} y$ is false or equivalently, $I^{x}(y)>I^{y}(x)$ is false. This proves that for each $x \in X$, $I^{y}(x) \geqslant I^{x}(y)$, i.e., $y$ is score undominated.

\subsection{The choice of optimal alternatives: the general case}

In order to focus our general discussion about optimality, we restrict our attention to the strict semantics of softarisons. Thus in this section all softarisons are asymmetric.

Ideally, a softarison might identify an alternative that irrespective of what attribute we look at, it is never beaten by any other alternative. We call any such alternative $E$ maximal:

Definition 4.11 We say that $y \in X$ is $E$-maximal for $\mathcal{S}$ if it is maximal for $R_{e}$, for each $e \in E$.

$E$-maximal alternatives should be quite rare. When $E$ is finite, they are easily identified by their inverse scores: they are the alternatives $o_{j} \in X$ that satisfy $I^{o_{i}}\left(o_{j}\right)=0$ for all $o_{i} \in X$. In practical terms, when a tabular representation exists, every row corresponding with $o_{j}$ should be filled with zeros, no matter which table we look at.

We can now observe that with this terminology, Proposition 4.5 can be expressed as in the following corollary:

Corollary 4.12 Fix any softarison over X, and suppose that $E$ is finite. Then all $E$-maximal elements are score undominated. 
In the very likely event that no $E$-maximal alternatives exist, we might at least recommend alternatives with the following property (which is weaker):

Definition 4.13 We say that $y \in X$ is pre-maximal for $\mathcal{S}$ if it is maximal for $R_{e}$, for some $e \in E$.

A pre-maximal alternative is such that for at least one attribute, it is never outperformed when we restrict our attention to that attribute. Nevertheless the existence of pre-maximal elements for a softarison is not guaranteed either.

When $E$ is finite, in between both concepts lies the following idea (i.e., it is weaker than $E$-maximal but stronger than pre-maximal):

Definition 4.14 We say that $y \in X$ is quasi-maximal for $\mathcal{S}$ if it is pre-maximal for $\mathcal{S}$ and the number of attributes $e$ for which $y$ is maximal of $R_{e}$ is the highest.

Definition 4.14 is relevant because when there are premaximal but not $E$-maximal alternatives, some pre-maximal alternatives are more acceptable than others. Alternatives that for example, are pre-maximal for all but one attribute, are preferable to those that are pre-maximal for only one attribute. When the number of attributes is finite, and they are equally important, then we can prioritize premaximal elements by the number of attributes for which no other alternative outperforms them.

Remark 4.15 It is also natural to generalize the concept introduced in Definition 4.14 to a 'weighted' version, provided that we have weights associated with the attributes in $E$. We just need to replace 'number of attributes' by 'total sum of weights of attributes' (quite like we did in Remark 4.15). We will not exhaust all theoretical possibilities of this problem of selection among pre-maximal elements here. The reader is addressed to [11] for inspiring antecedents in the literature about binary relations.

To conclude this section, we prove a theorem that links the quest for pre-maximal elements and soft topology. So, it bridges a gap for which excellent antecedents exist since Weierstrass extreme value theorem. We need the next new concept:

Definition 4.16 Suppose that $\tau$ is a soft topology on $X$. We say that a softarison over $X, \mathcal{S}=\left\{\left(F^{x}, E\right)\right\}_{x \in X}$, is $\tau$-continuous when $\left(F^{x}, E\right) \in \tau$ for each $\left(F^{x}, E\right) \in \mathcal{S}$. Put shortly: $\mathcal{S}$ is $\tau$-continuous when $\mathcal{S} \subseteq \tau$.

Theorem 4.17 Assume that $\tau$ is a soft topology on $X$ that is soft compact. Then for any pre-acyclic softarison $\mathcal{S}$ over $X$ that is $\tau$-continuous, there is a pre-maximal alternative.

Proof By absurdum, suppose that there are not pre-maximal alternatives for $\mathcal{S}$. Then for all $x \in X$, because $x$ is not pre-maximal, for each $e \in E$ there must exist $y \in X$ such that $x \in F^{y}(e)$. Therefore $\sqcup_{x \in X}\left(F^{x}, E\right)=\tilde{X}$, and each $\left(F^{x}, E\right)$ is soft open because $\mathcal{S}$ is $\tau$-continuous. In other words, the expression above assures that $\mathcal{S}$ is an open soft covering of $X$.

Soft compactness ensures the existence of a finite soft subcovering of $\left\{\left(F^{x}, E\right)\right\}_{x \in X}$, i.e., there are $\left\{x_{1}, \ldots, x_{n}\right\} \subseteq$ $X$ such that $\sqcup_{i=1}^{n}\left(F^{x_{i}}, E\right)=\tilde{X}$.

We shall produce a contradiction if we prove from the latter expression that each $R_{e}$ has a cycle, because $\mathcal{S}$ is preacyclic hence at least one relation $R_{e}$ is acyclic.

For any fixed $e \in E$, we have stated $\cup_{i=1}^{n} F^{x_{i}}(e)=X$. Consider $x_{1}$. There must exist $x_{i}$ such that $x_{1} \in F^{x_{i}}(e)$, i.e., $x_{i} R_{e} x_{1}$. If $i=1$, we have a cycle of $R_{e}$ (of length 1 ). Otherwise, we do not lose generality if we suppose $i=2$ (we can relabel the subindices of $x_{2}$ and $x_{i}$ since they are assigned arbitrarily) thus $x_{2} R_{e} x_{1}$. Now consider $x_{2}$. There must exist $x_{i}$ such that $x_{2} \in F^{x_{i}}(e)$, i.e., $x_{i} R_{e} x_{2}$. If $i=1$ or $i=2$, we have a cycle of $R_{e}$ (of length 1 or 2). Otherwise, we do not lose generality if we suppose $i=3$ thus $x_{3} R_{e} x_{2} R_{e} x_{1}$. We can proceed recursively until $x_{n-1} \in F^{x_{i}}(e)$, which produces either a cycle of $R_{e}$ or $x_{n} R_{e} x_{n-1} R_{e} \ldots R_{e} x_{2} R_{e} x_{1}$. But now because $x_{n} \in F^{x_{i}}(e)$ for some $i=1, \ldots, n$, we obtain a cycle of $R_{e}$. This completes the argument.

\section{A decision-making procedure based on softarisons}

In this section we adopt a procedural position. We set forth a decision-making algorithm for situations where a softarison is used in order to model the soft-theoretical comparisons between pairs of alternatives.

Our procedure is justified by the following argument. Alternatives that are score undominated, or weighted-score undominated when a vector of weights is available (Definition 4.3), are optimal choices. However these properties are very demanding, and we know that such alternatives may not exist (cf., Example 4.4). In their place, minimax alternatives are a nice substitute. To show why, we argue with the case where all attributes are equally important for the sake of clarity. When $I^{o_{i}}\left(o_{j}\right)=0$ for all $o_{i} \in X$, Proposition 4.5 guarantees that $o_{j}$ is score undominated. This is the most desirable case: no matter which attribute we look at, no alternative beats $o_{j}$. The same is true for the weighted case. It is very unlikely that this happens in practice. But we can first look at the maximum of $I^{o_{i}}\left(o_{j}\right)$ across $i$, i.e., $M_{j}=\max \left\{I^{o_{i}}\left(o_{j}\right) \mid i=1, \ldots, m, i \neq j\right\}$. This figure is the largest number of attributes for which there is some other alternative that outperforms $o_{j}$ in that number. So the smallest this figure is, the better. Under a cautious 
approach we should therefore recommend an alternative $o_{l}$ for which $M_{l}$ is smallest, i.e., minimize $M_{j}$ or find a solution for $\min _{j=1, \ldots, m} \max \left\{I^{o_{i}}\left(o_{j}\right) \mid i=1, \ldots, m, i \neq j\right\}$. Such an alternative is a minimax alternative.

We can therefore endorse the following procedure for decision-making: comparative performance with respect to $E=\left\{e_{1}, e_{2}, e_{3}, e_{4}, e_{5}\right\}$, where attribute $e_{1}$ refers to 'Common interview questions', $e_{2}$ refers to 'Role-playing exercises', $e_{3}$ refers to 'Practical tasks', $e_{4}$ refers to 'Case studies', and $e_{5}$ refers to 'Group presentations'. He/she adopts the weak semantics for the retrieval of information.

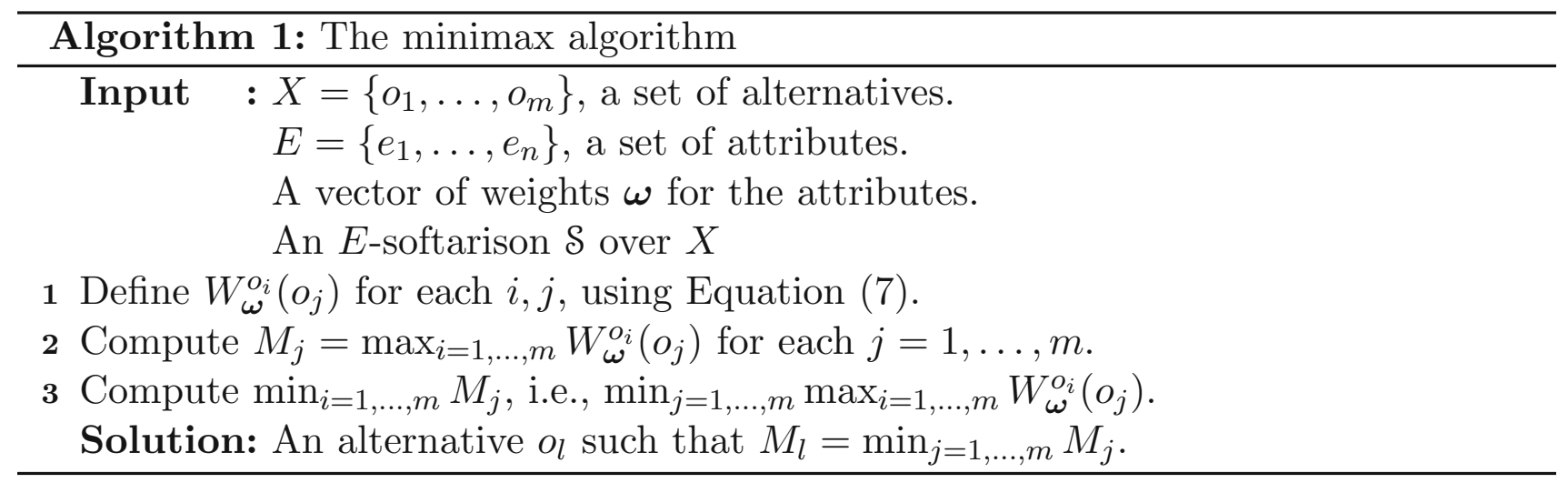

The figures $W_{\omega}^{o_{i}}\left(o_{j}\right)$ computed at Step 1 give us the overall importance of the attributes for which the other alternatives outperform $o_{j}$. Then, $M_{j}$ computed at Step 2 is a proxy for the worst possible comparison of $o_{j}$ versus any other alternative. Finally, we select an alternative that when compared with any other alternative, is outperformed in the least aggregate importance.

Remark 5.1 Some weighted-score undominated alternatives are detected by the minimax algorithm. Proposition 4.5 and its weighted counterpart assure that if $o_{j} \in X$ satisfies $I^{o_{i}}\left(o_{j}\right)=0$ (resp., $W_{\omega}^{o_{i}}\left(o_{j}\right)=0$ ) for all $o_{i} \in X$, then $o_{j}$ is score (resp., weighted-score) undominated. Notice that in this case $M_{j}=0$, therefore it minimizes $\left\{M_{1}, \ldots, M_{m}\right\}$ and this alternative will be selected by the minimax algorithm.

We apply the minimax algorithm to a practical casestudy in our next synthetic example, that concerns job hiring.

Example 5.2 Group interviews are an efficient interviewing technique whereby employers evaluate several candidates simultaneously in order to fill a position. It can also be used to hire more than one candidate for similar positions. Its main advantage is that it is less time-consuming than individual recruitment evaluations.

In this down-to-earth context, real data are highly confidential. Therefore the following streamlined situation is considered for illustration. Let $X=\left\{o_{1}, o_{2}, o_{3}, o_{4}\right\}$ be a set of four applicants. The employer considers their
After completion of the group interview, the employer produces a comparative performance of the candidates with respect to the activities in $E$. This generates a softarison $\mathcal{S}=\left\{\left(F^{o_{1}}, E\right),\left(F^{o_{2}}, E\right),\left(F^{o_{3}}, E\right),\left(F^{o_{4}}, E\right)\right\}$. Figure 7 displays such a softarison in tabular form. For illustration, the employer annotates 'When the common interview questions took place, I did not perceive that candidate $o_{1}$ performed at least as well as $o_{2}$ '. This annotation translates into $o_{2} \notin F^{o_{1}}\left(e_{1}\right)$, hence the 0 at row $o_{2}$ and column $e_{1}$ of the first table.

The attributes are not equally important. Their relative relevance is captured by a vector of weights $\omega=(0.2,0.2,0.3,0.15,0.15)$. Figure 8 shows the relevant $\omega$-weighted inverse scores associated with the softarison defined in this Example 5.2. These figures are defined at Step 1 of the minimax algorithm. The table shows the $M_{j}$ proxies defined at Step 2 of the minimax algorithm in its right column. The only solution is $o_{2}$, which is the candidate where the minimum of $M_{1}, M_{2}, M_{3}, M_{4}$ is attained.

Observe that the minimax algorithm has singled out a weighted-score undominated applicant: $W_{\omega}^{o_{2}}\left(o_{1}\right)=0.7$ $>W_{\omega}^{o_{1}}\left(o_{2}\right)=0.35, \quad W_{\omega}^{o_{2}}\left(o_{3}\right)=0.35=W_{\omega}^{o_{3}}\left(o_{2}\right), \quad$ and $W_{\omega}^{o_{2}}\left(o_{4}\right)=0.35=W_{\omega}^{o_{4}}\left(o_{2}\right)$. 


\begin{tabular}{cccccc}
$\left(F^{o_{1}}, E\right)$ & $e_{1}$ & $e_{2}$ & $e_{3}$ & $e_{4}$ & $e_{5}$ \\
\hline$o_{1}$ & 1 & 1 & 1 & 1 & 1 \\
$o_{2}$ & 0 & 1 & 0 & 1 & 0 \\
$o_{3}$ & 0 & 0 & 1 & 0 & 1 \\
$o_{4}$ & 1 & 0 & 1 & 1 & 0 \\
$\left(F^{o_{3}}, E\right)$ & $e_{1}$ & $e_{2}$ & $e_{3}$ & $e_{4}$ & $e_{5}$ \\
\hline$o_{1}$ & 0 & 1 & 1 & 1 & 0 \\
$o_{2}$ & 0 & 1 & 0 & 1 & 0 \\
$o_{3}$ & 1 & 1 & 1 & 1 & 1 \\
$o_{4}$ & 1 & 0 & 1 & 0 & 1
\end{tabular}

\begin{tabular}{cccccc}
$\left(F^{o_{2}}, E\right)$ & $e_{1}$ & $e_{2}$ & $e_{3}$ & $e_{4}$ & $e_{5}$ \\
\hline$o_{1}$ & 1 & 1 & 0 & 1 & 1 \\
$o_{2}$ & 1 & 1 & 1 & 1 & 1 \\
$o_{3}$ & 1 & 0 & 0 & 0 & 1 \\
$o_{4}$ & 1 & 0 & 0 & 1 & 0 \\
$\left(F^{o_{4}}, E\right)$ & $e_{1}$ & $e_{2}$ & $e_{3}$ & $e_{4}$ & $e_{5}$ \\
\hline$o_{1}$ & 1 & 0 & 1 & 0 & 1 \\
$o_{2}$ & 1 & 0 & 0 & 1 & 0 \\
$o_{3}$ & 0 & 0 & 1 & 0 & 1 \\
$o_{4}$ & 1 & 1 & 1 & 1 & 1
\end{tabular}

Fig. 7 Tabular representation of the softarison $\mathcal{S}=\left\{\left(F^{o_{1}}, E\right),\left(F^{o_{2}}, E\right),\left(F^{o_{3}}, E\right),\left(F^{o_{4}}, E\right)\right\}$ over $X$ in Example 5.2

\begin{tabular}{c|cccc|c} 
& $o_{1}$ & $o_{2}$ & $o_{3}$ & $o_{4}$ & $M_{j}$ \\
\hline$o_{1}$ & - & $W_{\boldsymbol{\omega}}^{o_{2}}\left(o_{1}\right)=0.7$ & $W_{\boldsymbol{\omega}}^{o_{3}}\left(o_{1}\right)=0.65$ & $W_{\boldsymbol{\omega}}^{o_{4}}\left(o_{1}\right)=0.65$ & 0.7 \\
$o_{2}$ & $W_{\boldsymbol{\omega}}^{o_{1}}\left(o_{2}\right)=0.35$ & - & $W_{\boldsymbol{\omega}}^{o_{3}}\left(o_{2}\right)=0.35$ & $W_{\boldsymbol{\omega}}^{o_{4}}\left(o_{2}\right)=0.35$ & $\mathbf{0 . 3 5}$ \\
$o_{3}$ & $W_{\boldsymbol{\omega}}^{o_{1}}\left(o_{3}\right)=0.45$ & $W_{\boldsymbol{\omega}}^{o_{2}}\left(o_{3}\right)=0.35$ & - & $W_{\boldsymbol{\omega}}^{o_{4}}\left(o_{3}\right)=0.45$ & 0.45 \\
$o_{4}$ & $W_{\boldsymbol{\omega}}^{o_{1}}\left(o_{4}\right)=0.65$ & $W_{\boldsymbol{\omega}}^{o_{2}}\left(o_{4}\right)=0.35$ & $W_{\boldsymbol{\omega}}^{o_{3}}\left(o_{4}\right)=0.65$ & - & 0.65
\end{tabular}

Fig. 8 Inverse scores of the softarison $\mathcal{S}$ defined in Example 5.2

\section{Discussion and conclusion}

Softarisons constitute a novel approach to the specification of comparisons among alternatives in the setting of soft set theory. It is valid when there are multiple features and we need to pay attention to them separately; however, we cannot assess the value of the alternatives. Instead, we can only provide a parameterized description of which alternatives each alternative improves upon. Soft relations [15] which had been studied in the literature originate with a very different motivation. Their structure and semantics are unrelated to ours.

Our main target in this paper is to show that softarisons facilitate theoretical manipulations in novel directions. In particular, with the help of the model presented here we have first established a close link between soft topology and the selection of 'best' alternatives in an infinite context. We have done this through the theoretical approach in Sect. 4.3. Hence one can regard this achievement as a practical application of softarisons to the expanding field of soft set topology. Admittedly, we have prioritized mathematical applicability to validation through examples. Nevertheless Sect. 5 has shown the sort of practical situations where softarisons provide the right conceptual model for decision-making.
Multiple lines of research open up and we have only settled the ground for some of them. Among the possible avenues for additional research we cite but a few:

1. One can still delve deeper into the debate procedural vs. normative solutions. Other procedural solutions can be produced and compared with our minimax algorithm.

2. In addition to the brief introduction in Sect. 3.3, much more can be said about the consistency properties of softarisons. Notice that the inspirational case of binary relations is enormously rich [18]. Procedures for their easy identification from the tabular form should be helpful in applications. Computer-assisted algorithms may be especially useful for large sets of data.

3. Multi-agent decision-making would require the utilization of several softarisons. Suitable solution concepts and mechanisms should be given accordingly.

4. A more general model (maybe called $N$-softarisons) can be defined with the assistance of $N$-soft sets [20]. They should be useful when we have multinary parameterized descriptions (of which alternatives each one improves upon) at our disposal. A full more general theory can be developed from this starting point. One could even resort to more general models for inspiration, like hesitant fuzzy $N$-soft sets [1]. Nevertheless, it is important that we investigate the bounds of the model by softarisons separately. 
Acknowledgements The author is grateful to the Junta de Castilla y León and the European Regional Development Fund (Grant CLU2019-03) for the financial support to the Research Unit of Excellence 'Economic Management for Sustainability' (GECOS).

Funding Open Access funding provided thanks to the CRUE-CSIC agreement with Springer Nature.

\section{Declarations}

Conflict of interest The author declares no conflict of interest.

Open Access This article is licensed under a Creative Commons Attribution 4.0 International License, which permits use, sharing, adaptation, distribution and reproduction in any medium or format, as long as you give appropriate credit to the original author(s) and the source, provide a link to the Creative Commons licence, and indicate if changes were made. The images or other third party material in this article are included in the article's Creative Commons licence, unless indicated otherwise in a credit line to the material. If material is not included in the article's Creative Commons licence and your intended use is not permitted by statutory regulation or exceeds the permitted use, you will need to obtain permission directly from the copyright holder. To view a copy of this licence, visit http://creativecommons. org/licenses/by/4.0/.

\section{References}

1. Akram M, Adeel A, Alcantud JCR (2019) Hesitant fuzzy $N$-soft sets. J Intel Fuzzy Syst 36(6):6113-6127

2. Al-shami TM (2021) Compactness on soft topological ordered spaces and its application on the information system. J Math 2021:6699092. https://doi.org/10.1155/2021/6699092. https:// www.hindawi.com/journals/jmath/2021/6699092/

3. Al-shami TM, El-Shafei ME (2020) Partial belong relation on soft separation axioms and decision-making problem, two birds with one stone. Soft Comput 24(7):5377-5387. https://doi.org/10. 1007/s00500-019-04295-7

4. Al-shami T.M, El-Shafei M.E, Abo-Elhamayel M (2018) Almost soft compact and approximately soft Lindelöf spaces. J Taibah Univ Sci 12(5):620-630. https://doi.org/10.1080/16583655.2018. 1513701

5. Al-shami TM, El-Shafei ME, Abo-Elhamayel M (2019) On soft topological ordered spaces. J King Saud Univ Sci 31(4):556-566. https://doi.org/10.1016/j.jksus.2018.06.005. https://www.science direct.com/science/article/pii/S1018364717313010

6. Al-shami TM, Kočinaç LDR, Asaad BA (2020) Sum of soft topological spaces. Mathematics 8(6):990. https://doi.org/10. 3390/math8060990. https://www.mdpi.com/2227-7390/8/6/990

7. Alcantud JCR (1999) Topological properties of spaces ordered by preferences. Int J Math Math Sci 22(1):17-27. https://doi.org/10. 1155/S0161171299220170. https://www.hindawi.com/journals/ ijmms/1999/329258/

8. Alcantud JCR (2002) Characterization of the existence of maximal elements of acyclic relations. Econ Theor 19(2):407-416. https://doi.org/10.1007/PL00004219

9. Alcantud JCR (2016) Some formal relationships among soft sets, fuzzy sets, and their extensions. Int J Approximate Reasoning 68:45-53

10. Alcantud JCR (2020) Soft open bases and a novel construction of soft topologies from bases for topologies. Mathematics 8(5):672
11. Alcantud JCR, Bosi G, Zuanon M (2010) A selection of maximal elements under non-transitive indifferences. J Math Psychol 54(6):481-484

12. Aleskerov F, Bouyssou D, Monjardet B (2007) Utility maximization choice and preference. Springer, Berlin Heidelberg

13. Ali MI, Feng F, Liu XY, Min WK, Shabir M (2009) On some new operations in soft set theory. Comput Math Appl 57(9):1547-1553

14. Aygünoğlu A, Aygün H (2012) Some notes on soft topological spaces. Neural Comput Appl 21(1):113-119. https://doi.org/10. 1007/s00521-011-0722-3

15. Babitha K, Sunil J (2010) Soft set relations and functions. Comput Math Appl 60(7):1840-1849. https://doi.org/10.1016/j. camwa.2010.07.014. http://www.sciencedirect.com/science/arti cle/pii/S0898122110004906

16. Babitha KV, John SJ (2013) Hesitant fuzzy soft sets. J New Results Sci 3:98-107

17. Bergstrom TC (1975) Maximal elements of acyclic relations on compact sets. J Econ Theory 10:403-404

18. Bossert W, Suzumura K (2010) Consistency choice and rationality. Harvard University Press, Cambridge, Massachusetts

19. Çağman N, Karataş S, Enginoglu S (2011) Soft topology. Comput Math Appl 62(1):351-358. https://doi.org/10.1016/j.camwa. 2011.05.016. http://www.sciencedirect.com/science/article/pii/ S0898122111004044

20. Fatimah F, Rosadi D, Hakim RBF, Alcantud JCR (2017) $N$-soft sets and their decision making algorithms. Soft Comput. https:// doi.org/10.1007/s00500-017-2838-6

21. Fatimah F, Rosadi D, Hakim RF, Alcantud JCR (2017) Probabilistic soft sets and dual probabilistic soft sets in decisionmaking. Neural Comput Appl. https://doi.org/10.1007/s00521017-3011-y

22. Feng F, Li Y (2013) Soft subsets and soft product operations. Inf Sci 232:44-57

23. Feng F, Liu X, Leoreanu-Fotea V, Jun YB (2011) Soft sets and soft rough sets. Inf Sci 181(6):1125-1137. https://doi.org/10. 1016/j.ins.2010.11.004

24. Han BH, Li Y, Liu J, Geng S, Li H (2014) Elicitation criterions for restricted intersection of two incomplete soft sets. Knowl Based Syst 59:121-131

25. John SJ (2021) Soft sets: theory and applications. Studies in fuzziness and soft computing, vol 400. Springer, New York

26. Liu XY, Feng F, Jun YB (2012) A note on generalized soft equal relations. Comput Math Appl 64:572-578

27. Liu Z, Alcantud JCR, Qin K, Pei Z (2019) The relationship between soft sets and fuzzy sets and its application. J Intell Fuzzy Syst 36(4):3751-3764

28. Ma X, Liu Q, Zhan J (2017) A survey of decision making methods based on certain hybrid soft set models. Artif Intell Rev 47(4):507-530

29. Maji P, Biswas R, Roy A (2001) Fuzzy soft sets. J Fuzzy Math 9:589-602

30. Maji P, Biswas R, Roy A (2003) Soft set theory. Comput Math Appl 45:555-562

31. Martínez-Legaz JE (2014) On Weierstrass extreme value theorem. Optim Lett 8(1):391-393. https://doi.org/10.1007/s11590012-0587-0

32. Mathew TJ, Sherly E, Alcantud JCR (2018) A multimodal adaptive approach on soft set based diagnostic risk prediction system. J Intel Fuzzy Syst 34(3):1609-1618. https://doi.org/10. 3233/JIFS-169455. https://content.iospress.com/articles/journalof-intelligent-and-fuzzy-systems/ifs 169455

33. Molodtsov D (1999) Soft set theory: first results. Comput Math Appl 37:19-31

34. Nachbin L (1965) Topology and order. D. Van Nostrand Inc., Princeton, New Jersey 
35. Qin H, Ma X, Zain JM, Herawan T (2012) A novel soft set approach in selecting clustering attribute. Knowl Based Syst 36:139-145

36. Qin K, Hong Z (2010) On soft equality. J Comput Appl Math 234(5):1347-1355. https://doi.org/10.1016/j.cam.2010.02. 028.http://www.sciencedirect.com/science/article/pii/ S0377042710001263

37. Shabir M, Naz M (2011) On soft topological spaces. Comput Math Appl 61(7):1786-1799. https://doi.org/10.1016/j.camwa. 2011.02.006. http://www.sciencedirect.com/science/article/pii/ S0898122111000927

38. Suzumura K (1983) Rational choice, collective decisions, and social welfare. Cambridge University Press, Cambridge

39. Terepeta M (2019) On separating axioms and similarity of soft topological spaces. Soft Comput 23(3):1049-1057. https://doi. org/10.1007/s00500-017-2824-Z

40. Walker M (1977) On the existence of maximal elements. J Econ Theory 16(2):470-474

41. Xiao Z, Gong K, Zou Y (2009) A combined forecasting approach based on fuzzy soft sets. J Comput Appl Math 228(1):326-333. https://doi.org/10.1016/j.cam.2008.09.033. http://www.science direct.com/science/article/pii/S0377042708005001

42. Xu W, Ma J, Wang S, Hao G (2010) Vague soft sets and their properties. Comput Math Appl 59(2):787-794. https://doi.org/10. 1016/j.camwa.2009.10.015. http://www.sciencedirect.com/sci ence/article/pii/S0898122109007147

43. Zhan J, Zhu K (2015) Reviews on decision making methods based on (fuzzy) soft sets and rough soft sets. J Intell Fuzzy Syst 29:1169-1176

44. Zorlutuna I, Akdag M, Min WK, Atmaca S (2012) Remarks on soft topological spaces. Ann Fuzzy Math Inform 3:171-185

45. Zou Y, Xiao Z (2008) Data analysis approaches of soft sets under incomplete information. Knowl Based Syst 21(8):941-945. https://doi.org/10.1016/j.knosys.2008.04.004

Publisher's Note Springer Nature remains neutral with regard to jurisdictional claims in published maps and institutional affiliations. 